\title{
Application of E-Learning Standardization Technology
}

\author{
Li Zheng, Lei Xu and Yushan Li \\ Tsinghua University \\ China
}

\section{E-Learning Standards in China}

\subsection{Standard Organizations}

With its rapid development, e-learning had a lot of problems, such as the resource formats not unified, lacks of unified interaction interface between learning platforms, interoperability between platforms not realized and so on. In order to solve these problems, a number of international organizations, as well as companies, are dedicated to the research of standardization. The most important organization include IEEE LTSC - Learning Technology Standards Committee• ; IMS• łMS Global Learning Consortium• of ISO and organization of National Educational Technology Standards for Students from different countries.

China E-Learning Technology Standardization Committee (CELTSC), a subcommittee under Standardization Administration of the People's Republic of China (SAC), is the research institute and management organization for standards development, as well as associated API and application system models.

Moreover, CELTSC, as the counterpart of ISO/IEC JTC1 SC36 in China, plays an important role in the international standards development and promotes the application and localization of those standards.

\section{2 CELTS Standards}

China had been basically formed a relatively complete standards system with the development over the past few years. The research work of standards can be divided into five categories: fundamental guidance, learning resources, learners, learning environment and education management. Some of these five categories are adapted or brought from IMS or IEEE, such as Architecture and Reference standard mainly refers to LTSA from IEEE. We can solve part of the problem of unification of platforms and resources through these five categories of standards.

Among these standards, "Architecture and Reference" is the most basic one. It provides the platform framework of e-learning, which describes the design and components of high-level system. 
The most widely used standards are Learning Object Metadata (LOM), Content Packaging• • $\mathrm{CP} \cdot$ and Learner Model. LOM is used to describe the information of learning object, aiming to help learners, educators or automated software to search, estimate, acquire and use the learning object. And learning object can exchange and use cross platforms through metadata model defined by LOM.

In CP standard, a data structure is defined to exchange leaning contents, in order to exchange and use learning contents in different creation tools, leaning management systems and process environments.

Learner model standard mainly formulate the semantics and syntax of learners information. Through learners data model, content management and learning managment system, leaner model can provide a more personalized and effective learning experience. Learners database created by this standard could be used in their whole education, learning experience and career path, and can be transplanted among different platforms.

Culture, belief and concept of a nation varies from nation to nation, which should be considered when develop e-learning service. And in China, some other typical factors should be noticed: political factors, individual file information, education background and management, and etc.

We make some rules based on local factors when we develop learners' model standards: we use residents' ID card to mark their identities. When we use vocabulary to describe learners' information during the LOM standard making process, we make vocabulary types based on domestic situation in China, such as "learning resource type" components vary widely from IEEE's LOM standard.

\section{The Design of Learning System Architecture}

Standard-setting is only a part of the standardization work. The promotion of these standards is more important. The application of standards is based on an open, standardized learning platform. We design a learning system structure based on "System Architecture "standard and " Learning system architecture and service interface " standard.

\subsection{Concept Model}

"System Architecture" standard provides high-level system structure of e-learning platform. This standard is based on LTSA of IEEE, as shown in Figure 1.

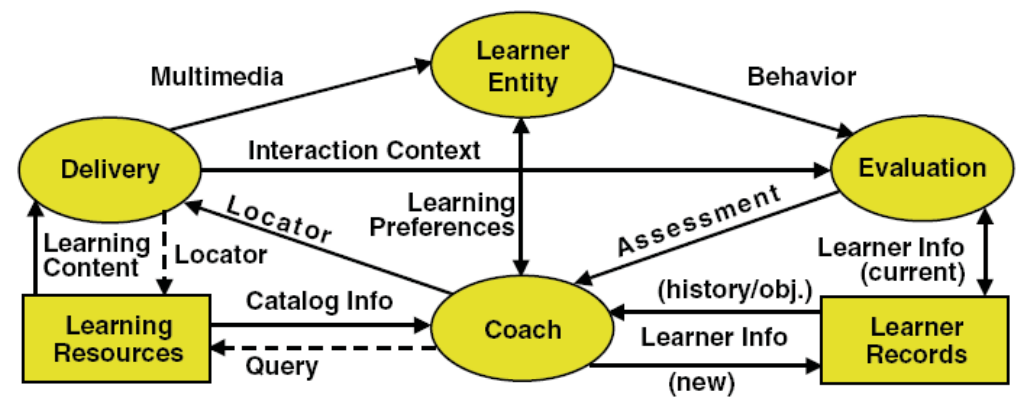

Fig. 1. Learning Technology Systems Architecture from IEEE 
In Figure 1, the system components are divided into three categories, namely:

1. (Processes): Learner Entity, Evaluation, Coach, Delivery

2. (Stores): Learner Records, Learning Resources

3. (Flows): Learning Parameters, Behaviour, Interaction Context, Learner Information, Assessment, Query, Catalog Information, Locator, Learning Content, Multimedia.

This architecture, describing the design and components of high-level architecture, is education irrelevant, content irrelevant, culture irrelevant and platform irrelevant. We have adopted architecture model as concept model of learning system. Based on this concept model, we have designed abstract framework and core interface.

\subsection{Abstract framework}

Based on the System Architecture standard, Learning system architecture and service interface standard design the architecture and service interface of learning system, as shown in Figure 2.

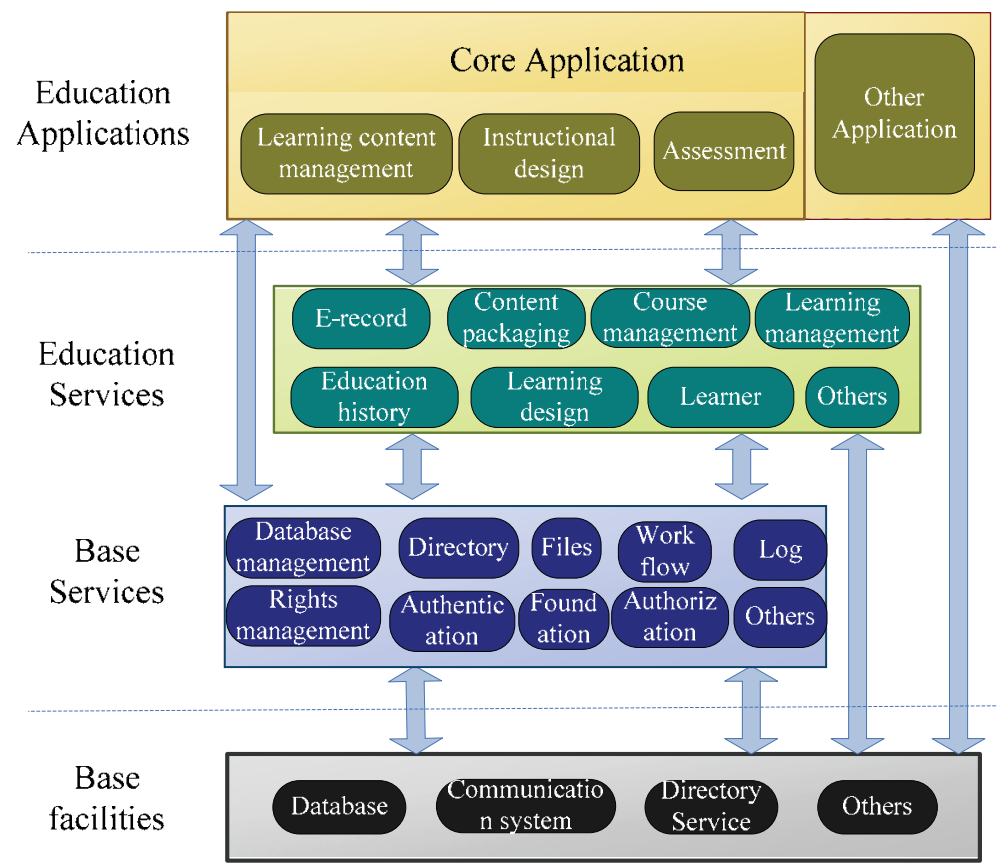

Fig. 2. Digital education leaning platform system layered architecture

a) Infrastructure layer: provide end-to-end transaction processing and communications services for education service and infrastructure service, including a variety of hardware and software, such as database management system, operating system, directory servers, application servers, network environment, etc.

b) Basic service layer: is integrated service could be used education service rather than digital educational platform. Basic service could use other basic service, so one service could be used in any other services. 
c) education service layer: provide core education application with integrated services necessary for digital educational platforms. A core education could use one or more basic services, which might use infratructure functions cross layers. Distributed education services communicate through infrastructure.

d) education application layer: this layer is some core applications and other applications, which are used in proper education service and presented to users, such as learning content management system, evaluation system. Appication could use apply one or more education service, and could use infrastructure service cross layer when neccesary. As to other applications, which could use necessary services in infrastructure, but the composition of system should be hidden to the users anytime.

UML diagram shows the relation among these layers:

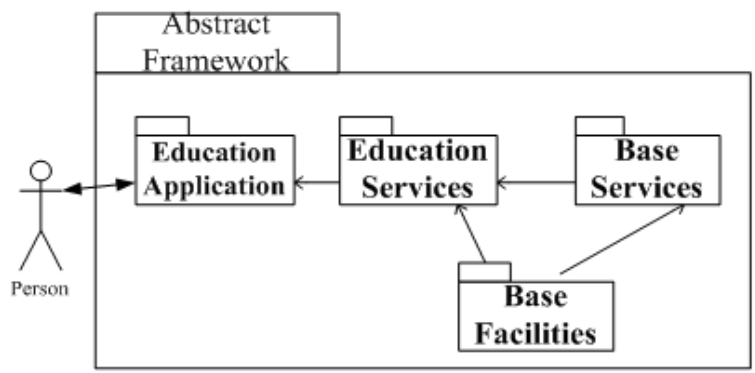

Fig. 3. UML graph

From the above layer structure, we find that this learning platform system reflects the hierarchical system, as well as service-oriented design principles. And the third education service layer supports the present e-learning recource standard, such as content packaging, learning design services, they all support CELTS and IMS standards.

One of the system architecture design principles is adopting abstract services to describe proper e-learning system function. One service is used to complete one functional requirement, that is, package a logical unit, service is the basic unit constructing educational application software. After the service is hidden in the service access point (ASP), and could only be accessed through SAP, API could be one way of accessing SAP, such as Java API. The services of e-learning architecture could be described as followings:

\section{Service ID}

SAP

SAP Definition
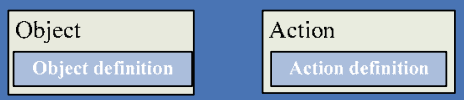

Implementation of private services

Fig. 4. abstract service 
Service has clear defined SAP, and each service has and only has one SAP. SAP in accordance with its composition of behavior and object should be defined. SAP include one or more object, and each object usually has more than one operation. This means that each service (including education service and public service) has to use this abstract service to define. In most cases, services will interact with each other, such as an education service uses public service. This interactive service is that service calls the SAP of required service.

\subsection{Core Interface}

In order to facilitate the reuse of services, we design standard interfaces for the core services. a) Education services layer

For an education system, education service layers have educational standard support, that is, this layer each service is generally required to follow corresponding standards. Such as content package service needs to follow the content package standard. For the educational service layer, it should include the followings:

1. Content package service: in view of standard content package, this service manages all the learning content with unified format, including delivery, reading, analysis, packaging and other functions.

2. Course management services: responsible for the management of curriculum-related content, including the creation of curricula, courses storage, query, display, and etc., as well as import and export of courses.

3. Members management service: provide members management-relevant function. In abroad sense, an individual member or a group can be called as member, and a group could be member of another group. And member information describes member role.

4. Packet Services: mainly related to the system packet management function. This service could be used to group members, and also could be used to group learning resources. So grouping is managed as a independent service rather than as a special case of member, which could give grouping behavior more space during the learning process.

5. Testing service: in view of a certain learning objectives, measure learners' learning results, which including generation, storage, retrieval of test questions, as well as organization and evaluation of test.

6. Instruction design service: provide generation, display, analysis and retrieval of instruction design services. Instruction design service is based on corresponding norms, and support the norms.

7. Sequencing service: encapsulate operation and data model which is related with sequencing activity. CELTS, IMS and SCORM all have the sequencing standard, to meet the different standards' need, sequencing service should provide sequencing rule model, sequencing activity, sequencing engine and sequencing analyze interfaces.

b) Basic service layer

Basic service layer need to provide functions commonly required by education service layer. The design of these services is based on education service layer, but the result of the design is not only limited to support of education service. The basic service layer includes:

1. Certification service: this service is used to obtain certification information from users, confirm the authenticity of users' identity, and allow users to log on the system. 
Other services or application could check or operate the status of users' certification, ignoring the details of certification process. This service is built on all kinds of certification establishment. Many agencies have already had or are trying to build their own center certification. Technically speaking, we have examples like Kerberos, X.509, and single-point embedding technology based on cookie, such as webISO.

2. Authorization service: this service provides service of building and querying authorization information for other services. Authorization information mainly provides a user the authorized information.

3. File operation service: this service provides operation interface for platformindependent file access.

4. Database service: this service provides database related access interface.

5. XML service: this service provides XML operation related interface, including XML analysis function. Since most semantic information of normative text can be bound to XML, XML service could further make XML mapping as object directly used by developers.

6. Digital rights management service: this service provides model related to authorization information used by learning object. And it also provides management function, including provision, control, tracking and management of copyright information.

7. Log service: this service provide log function, being used to track and record relevant information and events of other services. System maintenance and monitoring might need to use log. And log could also be used for performance analysis, data collection and statistics.

Based on the service layer relation and logic function analysis, we develope a complete set of interfaces for each service for the "core interface", and develope "Learning system architecture and service interface" standard, provide architecture design proposal for developers.

\section{Key Implementation Technology}

For a learning system, the most important part is learning resource management and learning process management. Next we will analyze the key implementation technology of these two parts.

\subsection{Supportive of Resource Sharing}

Learning resources management is the essential part of e-learning systems, by which we can describe, organize and share learning resources. There are many standards used to manage learning resources, such as LOM and CP standards. LOM standard is the foundation of learning resource management, it is mainly used to describing learning resources. $\mathrm{CP}$ standard can be used to organizing learning resources to be a knowledge unit or a course. The common target of these two standards is sharing learning resources, so we can exchange and use learning resources under different platforms. To achieve this target, we choose XML as primary binding format. 


\subsubsection{Persistence of XML}

$\mathrm{XML}$ is chosen as binding format by many e-learning standards, that is because of the good expansibility of XML, and as a kind of plain text format, we can process and transfer XML files easily.

Generally speaking, we can store XML files in three ways: file system, relational database (RDB) and XML database. XML files can be transplanted easily under file system, but searching an XML file is difficult. To be stored in RDB, XML content can be stored as long text format. And we can store some commonly used properties in a separate table in the database to improve searching speed. RDB is also easy for importing and exporting XML files. If XML database is used to store XML files, we can use XQuery to locate, update and delete corresponding XML files, and this method is the fastest and most convenient. XML database also has functions to import and export and has good transportability.

We also have to process XML files in programs, getting or storing elements content in XML files. To achieve this target, we choose Castor XML and JiBx tools for implementation. By these tools, we can marshal almost any "bean-like" Java Object to and from XML.

\subsubsection{Standard Transformation}

Different learning resource management systems are very likely based on different standards. For example, SCORM, Blackboard, Moodle and Sakai are mainly based on IEEE LOM standard to manage LOM objects. At the same time, many other systems are using similar standards, such as CELTS LOM standard or IMS MLR standard. Among all these standards, differences are inevitable, which makes it necessary to find ways to easily transform among similar standards. So we designed transformation tool to transform different LOM standards.

As a lot of standards have chosen XML as primary binding format, so the transformation between different standards is mainly transforming XML files, which can mapping one element in a standard file to another response element in other standard files. XSLT (Extensible Stylesheet Language Transformations) is adopted to transform among different metadata models. As described in Figure 5, we can convert the original CELTS LOM into IEEE LOM or MLR by XSLT Processor.

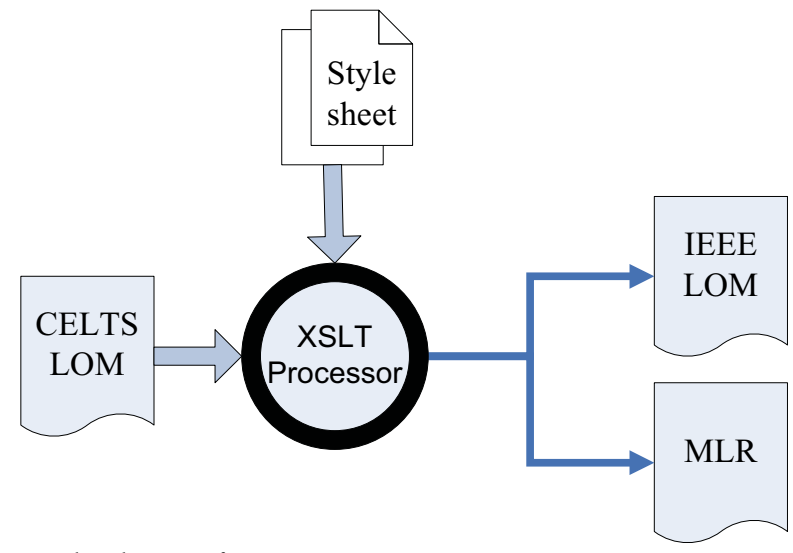

Fig. 5. Process of Standard Transformation 
XSLT is an XML-based language used for the transformation of XML documents into other $\mathrm{XML}$ documents. It relies upon the W3C's XPath language for identifying subsets of the source document tree. For example, if we convert "identifier" element in CELTS LOM standard to "ResourceIdentifier" element in IEEE LOM standard, we can use the following XSLT code.

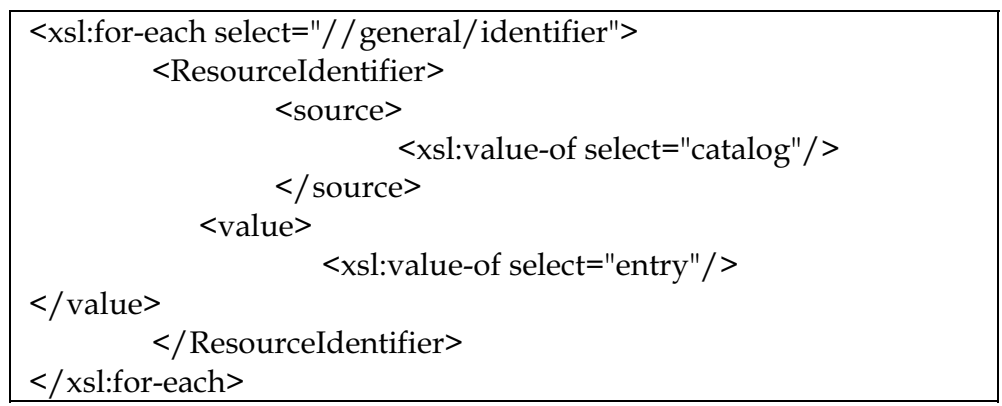

In the code, we can use "select" statement to query an XML element, and we can also fetch the element value by using "xsl:value-of" statement. Next step we can read pending XML file and XSLT file by java language, and with xalan package (supported by jdk1.4 version and above), we can get a MLR instance.

So in of the steps above, the most important step is finding mapping rules between different standards, and writing them into XSLT file, and finally we can use JAVA language to get the converted file.

\subsection{Supportive of Learning Process}

In an advanced learning system, users cannot only manage and share resources, but also can study and discuss. This needs learning system to be able to manage learning processes. The learning process here is not just reading materials or posting a question in a forum, but also collaborative learning, personalized learning process design, role plays and etc. For a learning system, we need to design functions to support the management of collaborative learning and personalized learning flows, and we also need to handle complicated elements in learning design.

\subsubsection{Collaborative Learning and Personalized Learning Flows}

For supplying a better learning experience, we focus on designing a human-based learning environment. We believe that if we involve the students to the process of creating, improving and populating instructional design, there will be a remarkable growth in their learning motive and enthusiasm which not only helps them to achieve better learning outcomes, but also the teachers to better adopt their design to learners' requirements. We mainly use collaborative learning and personalized learning flows to achieve this purpose. The base form of collaborative learning is learners working as a group to complete a learning activity. For a collaborative learning flow is mainly composed of the following parts: participator role, learning grouping (organization structure) and the information flow and transmission in the minute work. Compared to traditional e-learning, collaborative learning has introduced the concept of role. The enthusiasm of learners can be greatly 
improved by the rational allocation of roles, and in the process of collaborative learning, the supervision mechanism in the group can let learners put enough energy in learning activity. Personalized learning means satisfying individual requirements in the learning process, and improving learners' effect. When someone is studying, he may have such needs: raise up questions about unclear knowledge points, pointing out the errors in courseware, posting his experience about some knowledge point. Although there are some e-learning systems can meet these needs, users have to leave there studying interface, which means users' learning is interrupted. If we integrate all these functions in the learning interface, users' learning process won't be interrupted and users are encouraged in participating these activities. To meet these needs, the personalized learning flow is designed as below:

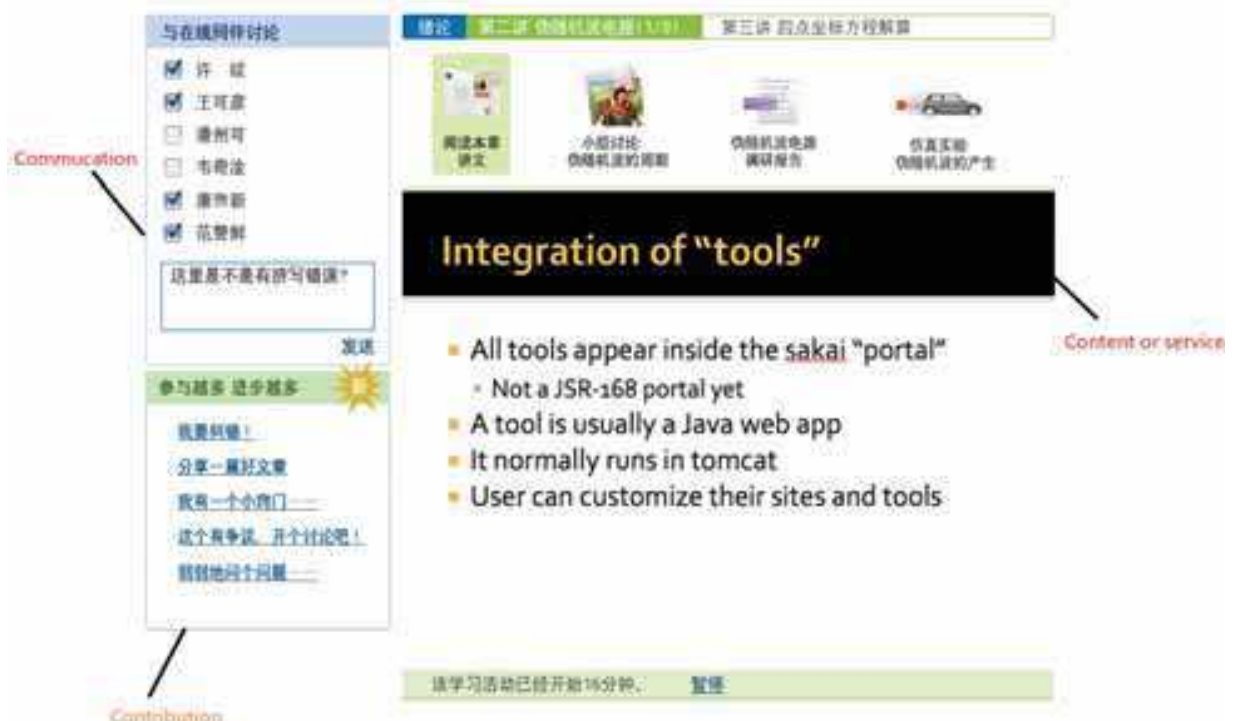

Fig. 6. Personalized learning flow design

\subsubsection{Handling Elements with Description Patterns}

When systems have to handle XML elements, they usually use castor tool to marshal java elements from and to XML elements, and then the system present the interface for users to edit or view, this method is sufficient for handling elements of fixed types. But in the process of editing learning design, the types of elements are not fixed. For example, an element can be "Boolean" type or "String" type, for the former type, the interface has to show a group of two radio buttons, and for "String" type, a text field has to be shown to users. So in the learning design platform, castor XML isn't enough for handling elements.

We propose to use description patterns to solve the problem above. Simply speaking, description pattern is used to describe an attribute whose type isn't fixed, and unify the front-end display of elements of numerous types. The process of handling elements with description patterns is shown as below: 


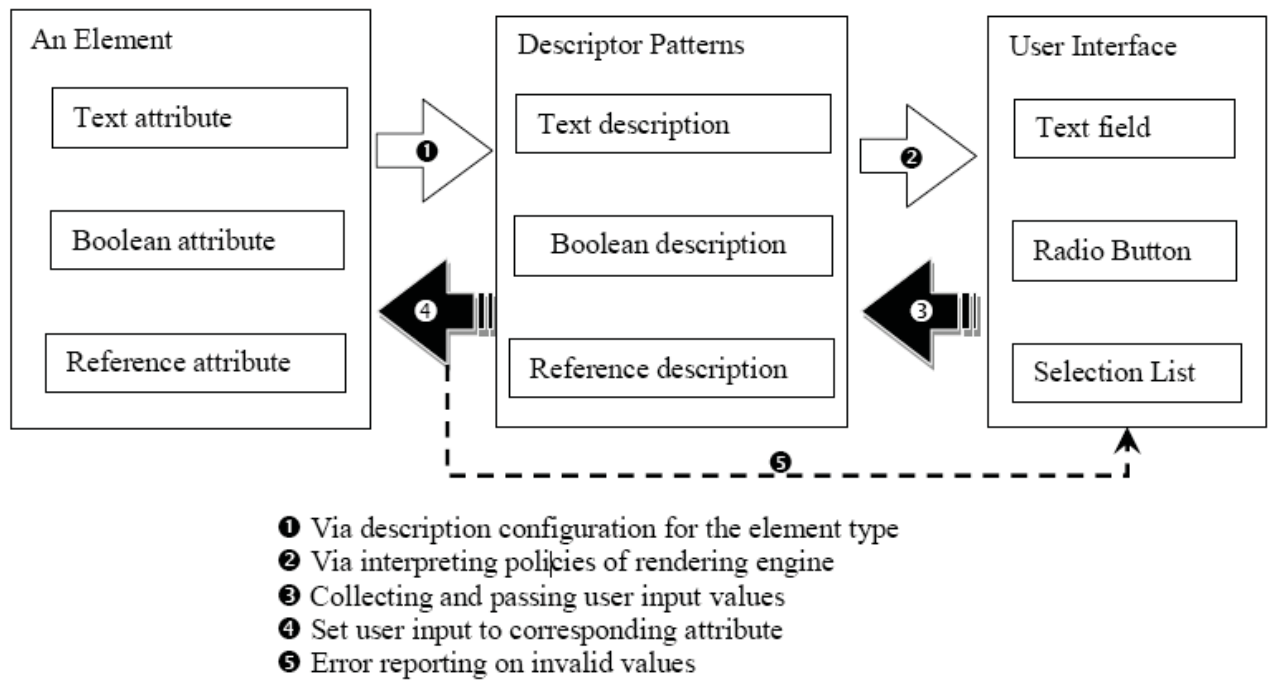

Fig. 7. Generating attribute sheet for an element

For a request element, the system first obtains the description pattern for it, and then it builds the input field for each pattern. For example, to present a text attribute description, the system may dynamically create a text filed in the property sheet. And for a Boolean description pattern, it creates a group of two radio buttons or a selection box with two options. After the author modifies some of the attributes, the system passes each modified attribute value to the corresponding description pattern and tells them to set the new values.

\section{LOM Editor}

LOM editor is designed and developed based on CELTS LOM standard. Since LOM standard is used to describe learning object information standard, LOM management tool is the most basic part of education system. LOM management system has the following functions:

1) Generate XML document meeting LOM standard based on data provided by users, and store in database.

2) Modify the specified XML file.

3) According to users' requirement, query specific LOM elements (such as title, author, and etc.) from database storing XML file.

During the developing process, we have applied RDB as a tool storing XML file, and chose JAVA as developing language. We first determine the implementation of underlying function:

1) Define all LOM elements by JAVA, for example, title of LOM is a multi-language string types, including language attributes, as well as string attributes. When using JAVA, definition includes title type with two attributes and some attribute operation functions.

2) Castor tool realizes the mapping from XML file to JAVA elements, which could allow elements in XML file exchange with corresponding JAVA elements in step 1. 
3) Define the interfaces interactive with database. In order to operate database more conveniently, we apply hibernate technology. The reasons for choosing hibernate are 1) system could replace database more easily. For example, we only need change configuration files, don't need to re-write code when replace database from Oracle to Mysql; 2) hibernate allows storage and retrieval without writing database operation sentences, could be easily developed.

4) Database table design. Besides design of a separate table for storing XML document, we put some frequently query attributes in the separate database table. These two tables connect through foreign key attribute of database. The reasons for storing attributes separately to one table is by this way the retrieval could be faster, otherwise, when query system need first convert XML file to JAVA elements, which wastes.

We can summarize the design steps by using the chart below:

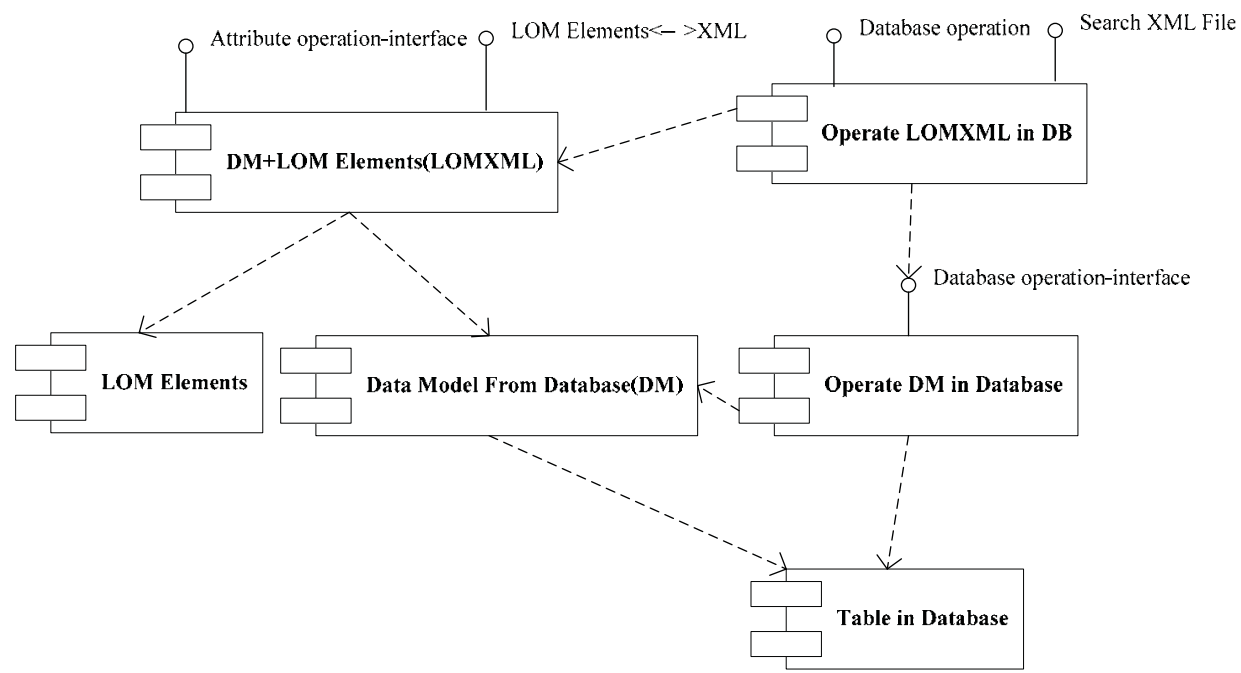

Fig. 8. LOM management tool underlying function design

Based on the above underlying function design, we have applies J2EE to realize the interaction between surface and under layer. The surface calls the under layer interface function through users operation. The editing interface of LOM is shown in the graph below, LOM object could be generated by filling table in the graph. 


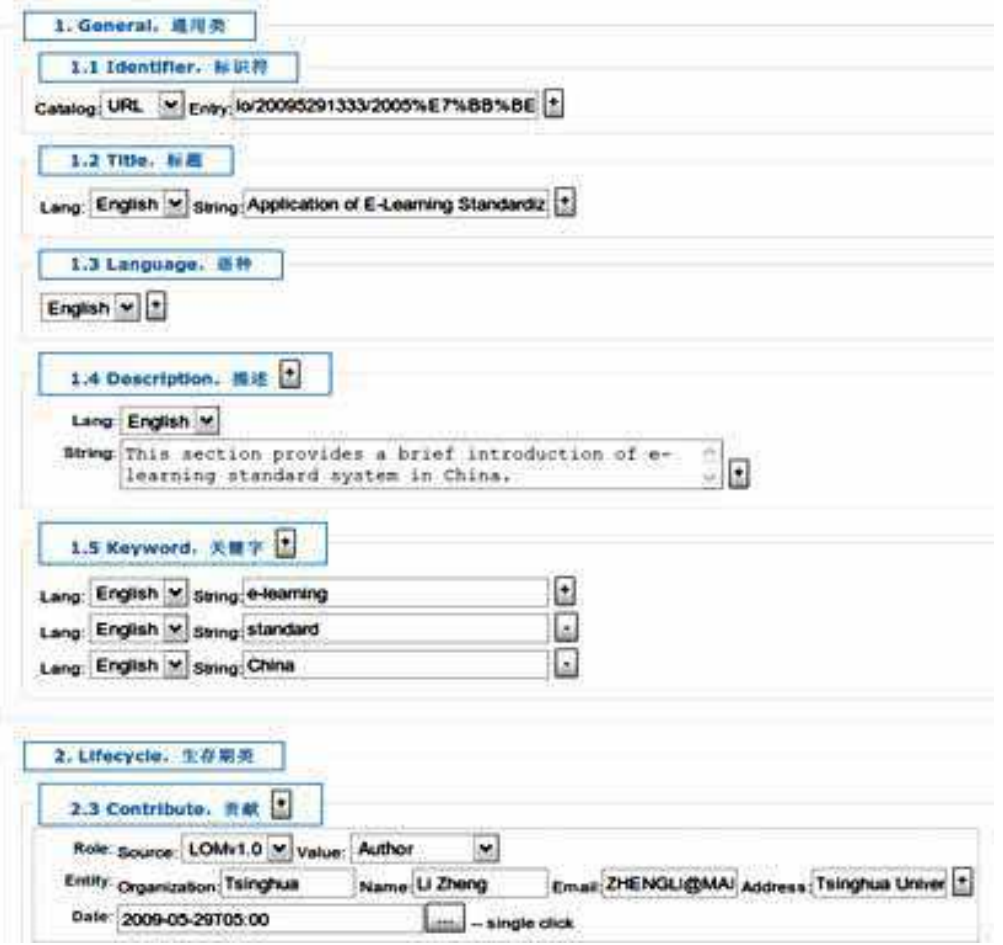

Fig. 9. LOM editor editing interface

\section{Content Package Management System}

Learning object, the smallest reusable unit in modern e-learning system, could be generated by LOM tool. Content package service is another education core service based on that. This service is designed and developed based on CELTS CP standard.

The function of content packaging is that it defines a unified data model, allowing learning resources could be organized randomly. This newly organized learning resources (here called learning content) could be copies, transferred, purchased, implemented and used independently, and could be further organized into bigger unit, which enhances the interoperability of learning resources and increases the efficiency of learning resource production.

Content list, the main part of content package management system, includes the following four parts:

1) meta-data components: overall description of content list.

2) Organization structure part: description of one or more means of leaning content organization from content list.

3) Resource reference part: reference of all the actual resources and all the necessary media resources in content list, and also include metadata describing resources and reference of external files.

4) The content list: zero or multi-nested content list. 
Content list combined with physical data construct a complete learning content. During this process, the most important part is the generation of content list. As LOM tool, learning content package tool, based on WEB, applies J2EE technology, and uses content package service interface API.

The content package list data uses XML format, stored in a relational database. The core of the system is similar to LOM tool, including two parts:

1) content package standard API: abstract data part of content package service including definitions of all involved data structures, corresponding XML operation interfaces realized through XML bounding norms based on content package and interactive interfaces of relational database system. For this part, the design of API is basically the same as API of LOM.

2) page logic control modules: process and control various operations of content package and make page logical respond, mainly used in functions like responding to users' add, edit, delete the node, and etc.
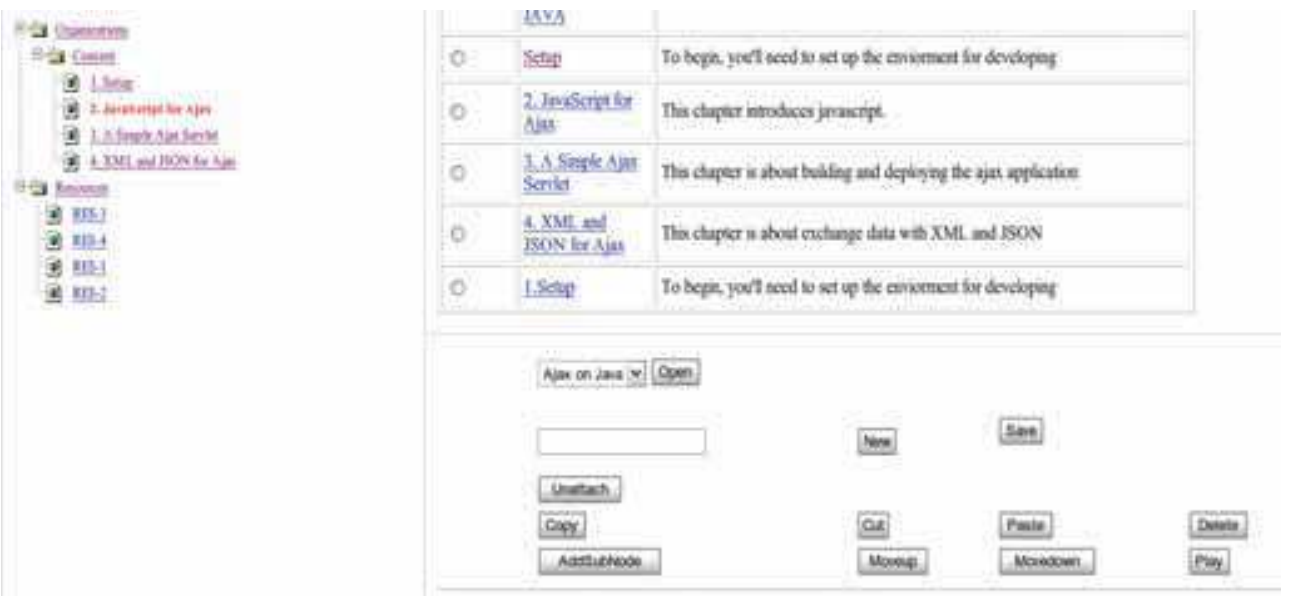

Fig. 10. the content package editing interface

System provides users with various editing function in view of content list (as shown in above graph), including:

1) Add sub-node: add a new sub-node under the current node.

2) Edit the node: change the name of the current node and the corresponding parameters.

3) Delete the node: delete the current node and the whole content under the current node.

4) Save: save content list as XML format and provide download link.

5) Upper and lower move node: change node order

6) Copy, paste: copy, paste nodes, including the contents under the node.

7) Play: play files described by the current node, such as HTML, Flash, PowerPoint, etc.

8) New: create a new content list.

In the process of editing, system would provide real-time content list view to users. After editing, users could browse the content list in XML format. 


\section{Learning design support system Information}

Support to learning design is the important base for learning process management. Leaning design including organization of education resources and also have learning activities and participants' role design. We have achieved the management of collaborative learning flow and personal leaning flow based on leaning design support.

\subsection{System layered architecture and components proposal}

Learning design platform could be an independent system, and also could be a bigger component of learning platform. So we need to make it not only could be exist independently, but also easily integrated. The whole platform is designed in accordance with "Learning system architecture and interface" standard, which is layered architecture, as shown in Figure 11, the dotted lines between services present call relation.

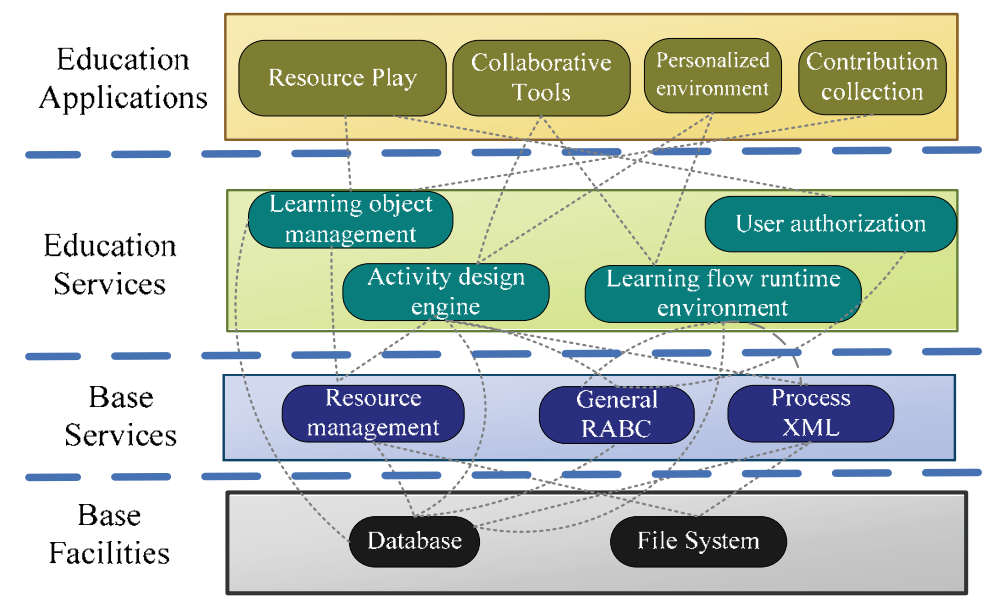

Fig. 11. Layered architecture design of learning design platform

From the above architecture, we find that in addition to specialized services like learning design, some other services, like LOM service, certification and authorization of participants, have been already exist. In order to promote reuse of e-learning service in different systems, we have adopted service-oriented approach to design all kinds of services of the above layered architecture.

\subsection{Data Exchange}

In the service-oriented system, either call service or service return results, they both need data exchange. In the service-oriented design, there are commonly two means of exchanging data.

1) Web Service and its description language WSDL, this service adopts XML as data exchange format, and provides a method of packaged service including registration, discovery, call and the combination. Since XML has poor support in HTML 
application environment, this method is not friendly to JavaScript, and could only obtain native support form high version Flash.

2) the way HTTP request +JSON, it applies JSON as data exchange format, high efficiency, which obtain good support in JavaScript and Flash, more practical, but lacks unified service description method, and when used alone could only through specific technical documents coordination.

From the above analysis, we find that Web Service form is more suitable loosely coupled systems, but JSON is more suitable for tightly coupled systems. We have proposed that the above two call means should be provided at the same time to a set of interfaces of one service. Since JOSN lacks unified service description method, we have adopted WSDL to describe HTTP request +JSON service. Compared with Web Service, except the different access points, other attributes (operation name and parameters) are all the same.

\subsection{Implementation proposal of front-end system}

Since the system need visual management of information entities, in the browser application, we have chose RIS (Rich Internet Application). Ajax and Flash are two technique routes of RIA. In this system, the main framework is achieved through Ajax framework Cappuccino, but the monitor dynamic graph is realized by Flash. And RIA applies asynchronous data transmission, just to meet the need of service-oriented data provision method. So these two technologies are very suitable for front-end system development.

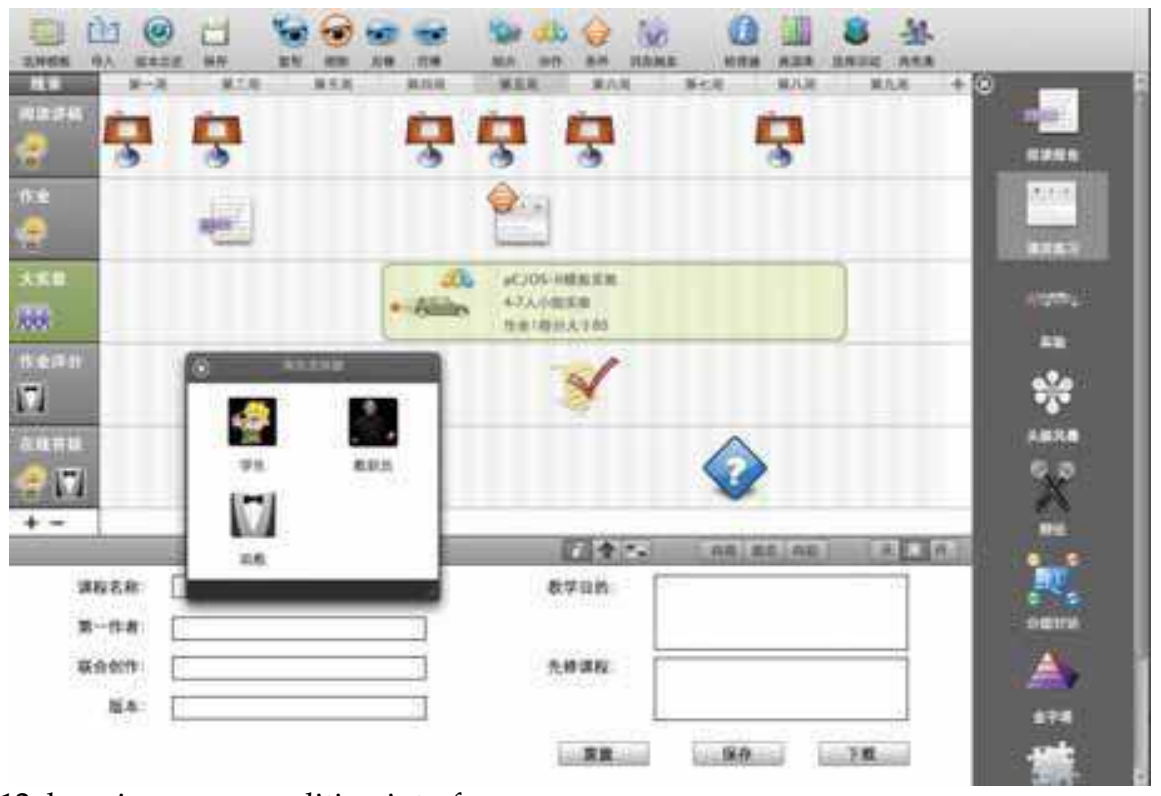

Fig. 12. learning process editing interface

Cappuccino framework could help developers build local-level application in browser, which is the ideal choice of developing relatively complicated deign and process 
environment in view of learning flow. The framework design Objective-J language, which could be mix-used with JavaScript, completing a challenging task to traditional Ajax. In addition to solve the complicated interface display, Cappuccino and Flash exchange data with Backend Server by using JSON, which increase the efficiency of data transmission, and achieve the greatest decoupling between front-end and backend server. For example, the editing interface of leaning flow is shown a Figure 12.

In the Figure 12, the right side has some default activities, including experiment, group discussion, and book report. The activity operation includes copy, delete, import and export, and each activity could be gave a role, and the dialog is the role selector. In the design process, users could edit the learning flow through drafting, and could create role and event according to their own need.

\section{Summary}

This chapter gives a brief introduction to e-learning standard situation and the implementation technology of standard. It focused on learning resources standardization and management technology, and learning process management technology based on learning design standard. The implementation technology is only a sample of Chinese elearning standard implementation. In different learning systems, there are different proposals for resources standardization. But effective management of personal learning process, based on instruction design standard, has not been implemented in various learning systems. The learning design support system is only an attempt.

\section{References}

CELTS - Chinese eLearning Technology Standard, http:/ / www.celtsc.edu.cn/

CELTS, Specification for Achitecture and Reference, CELTS-1, CD2.0, 2004.

CELTS, Specification for Content Pakaging, CELTS-9, CD2.0, 2004.

CELTS, Specification for Learner Model, CELTS-11, CD2.0, 2004.

CELTS, Specification for Learning Object Metadata, CELTS-3, FDIS, 2008.

CELTS, Specification for System Architecture and Interface, CELTS-43, WD2.0, 2007.

IEEE Learning Technology Standards Committee (LTSC). IEEE P1484.1/D9. Draft Standard for Learning Technology - Learning Technology Systems Architecture (LTSA), 2001.

Lei Xu, Li Zheng, Jing Liu, Yintao Liu, Fang Yang, Description Patterns in Learning Design Authoring Systems, Blended Learning, pp. 315-326, Pearson, 2007.

Lei $\mathrm{Xu}, \mathrm{Li}$ Zheng, The Design of a Social Instructional-Design Platform, 1stInternational Conference on Hybrid Learning-ICHL 2008, pp. 108-116, 2008, Hong Kong

Li Zheng, Yintao Liu, Jing Wang, Fang Yang. "Multiple Standards Compatible Learning Resource Management", ICALT 2008, Santander, Cantabria, Spain, 2008. 


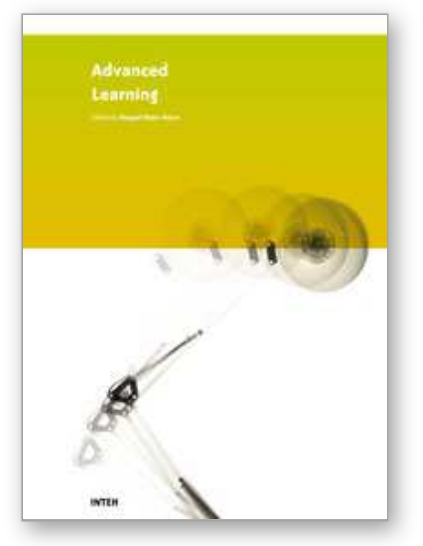

\author{
Advanced Learning \\ Edited by Raquel Hijn-Neira
}

ISBN 978-953-307-010-0

Hard cover, 444 pages

Publisher InTech

Published online 01, October, 2009

Published in print edition October, 2009

The education industry has obviously been influenced by the Internet revolution. Teaching and learning methods have changed significantly since the coming of the Web and it is very likely they will keep evolving many years to come thanks to it. A good example of this changing reality is the spectacular development of eLearning. In a more particular way, the Web 2.0 has offered to the teaching industry a set of tools and practices that are modifying the learning systems and knowledge transmission methods. Teachers and students can use these tools in a variety of ways aimed to the general purpose of promoting collaborative work. The editor would like to thank the authors, who have committed so much effort to the publication of this work. She is sure that this volume will certainly be of great help for students, teachers and researchers. This was, at least, the main aim of the authors.

\title{
How to reference
}

In order to correctly reference this scholarly work, feel free to copy and paste the following:

Li Zheng, Lei Xu and Yushan Li (2009). Application of E-Learning Standardization Technology, Advanced Learning, Raquel Hijn-Neira (Ed.), ISBN: 978-953-307-010-0, InTech, Available from:

http://www.intechopen.com/books/advanced-learning/application-of-e-learning-standardization-technology

\section{INTECH}

open science | open minds

\section{InTech Europe}

University Campus STeP Ri

Slavka Krautzeka 83/A

51000 Rijeka, Croatia

Phone: +385 (51) 770447

Fax: +385 (51) 686166

www.intechopen.com

\section{InTech China}

Unit 405, Office Block, Hotel Equatorial Shanghai

No.65, Yan An Road (West), Shanghai, 200040, China 中国上海市延安西路65号上海国际贵都大饭店办公楼 405 单元 Phone: +86-21-62489820

Fax: +86-21-62489821 
(C) 2009 The Author(s). Licensee IntechOpen. This chapter is distributed under the terms of the Creative Commons Attribution-NonCommercial-ShareAlike-3.0 License, which permits use, distribution and reproduction for non-commercial purposes, provided the original is properly cited and derivative works building on this content are distributed under the same license. 